\title{
Substantiation of methods of improving safety of pipeline gas transportation
}

\author{
O.A. Kurasov, P.V. Burkov
}

National Research Tomsk Polytechnic University, Division for Oil and Gas Engineering, postgraduate, Tomsk, Russia

\begin{abstract}
Ensuring the operational efficiency of hazardous production facilities throughout their entire service life becomes a formidable challenge associated with coordinating thorough monitoring of such facilities, assessing their exposure, mitigation and sustainability, and taking measures to minimize and prevent negative outcomes that can lead to negative consequences, including human fatalities, injuries and economic losses. At the same time, the optimal choice of the ratio between levels of risk and costs of identification, regulation and increase in the resource will ensure both a reduction to a minimum of the risk of failures in gas pipeline systems and increase the efficiency of resource use to maximize technogenic safety. The paper proposes a methodology for the calculation of risk indicators and the probability of emergencies on gas networks and gas distribution facilities during their operation in normal and emergency situations.
\end{abstract}

\section{Introduction}

Despite the mandatory protective measures taken to prevent emergency situations while ensuring an uninterrupted supply of natural gas to final consumers at the level of design parameters, accidents still occur. The more complicated the pipeline system, the industrial process, and the challenging natural environment are, the greater is the probability of an accident occurring over a certain future period. There also are low safety standards for data analysis of gas plant accidents.

Gas transportation systems are distinguished by the use of progressive and complex structural and technological solutions. The use of contemporary high-strength materials in their constructions allows operating them under the action and in extreme conditions of high levels of residual stresses, static, cyclic, and dynamic loads, in a wide range of temperatures and to be exposed to aggressive media.

Reliability of gas supply through pipeline systems is of utmost importance for meeting customer demand for gas. The consequences of a lack of gas supplies canbe catastrophic; hence a study of the reliability of these networks is important. Such study includes the following: maintaining the security of supply of gas, introducing steps to enhance the efficiency of the supply of gas, enhancing the economic and social benefits of pipeline companies, and optimizing operating and construction costs [1]. The focus of this study is therefore on the investigation of the reliability of the gas supply. 
Therefore, an accurate measurement of gas supply capacity and market demand, as well as consideration of their uncertainty, are crucial for determining the reliability of gas supply. However, most existing approaches neglect the complex behavior of the gas pipeline system when measuring gas supply capacity. In particular, high-pressure gas pipelines have significantly slower dynamics due to the large volume of natural gas contained in the pipelines, which are often referred to as line packs. The presence of a line pack is one of the most important distinctions between the gas pipeline system and other energy supply networks $[2,3,4]$.

Analysis of other research carried out in the field of reliability evaluation of pipeline networks has shown that these works present case studies of specific networks and, in most cases, the investigation focuses on the analysis of certain processes that are critical for reliability (such as degradation $[5,6,7]$ or available statistical data. There are several research works on pipeline system reliability assessment focused on failure data analysis [8] and structural integrity analysis [9].

They ought to take into consideration aspects of protection due to the large-scale danger of fire, explosion, and environmental contamination, given the impossibility of utterly removing the destruction of storage tanks and pipelines.

Quantitative risk analysis in gas pipelines and storage tanks for a mixture of natural gas and gas condensate is a tool for quantifying damage and planning measures to protect the exposed substance and people in surrounding areas. Determination of the zone of vulnerable damage based on the comparative characterization of known exposure levels can allow evaluating individual risk indicators for reaching the limit states according to the strength and resource parameters, which depend on the evolution of adverse situations. In emergency risk management and solving industrial safety problems, estimation of error potential is used only to consider random events with a high probability of occurrence. However, a quantitative-numerical determination of acceptable safety, risk, and security indicators is a priority [10].

It is important to analyze aging and recognize the key deterioration mechanisms in the systemic efficiency and risk analysis of energy supply networks. In the case of current district gas supply network depletion structures, it is important to conduct a strengthreview. In this review, the degradation of material properties and geometry data of pipelines should be analyzed. For intensity analysis of pipeline networks, loads from the hydraulic analysis can also be used [11].

Along with improving the operational characteristics of gas distribution networks, the safety requirements have also significantly increased. Appropriate attention should be given to the statistical and computer simulation of structural limitations during the operation of gas distribution networks under difficult conditions, taking into account both primary and secondary risk factors.

Pipelines are complex, dynamic structures. Leaks in gas pipelines can lead to explosions, fires and cause environmental, human, and material damage. Effective risk analysis is essential to prevent and reduce the probability of a possible accident. Timely measures taken to ensure the safe operation of gas stations can minimize their accident rate.

This article proposes design approaches for ensuring the technogenic safety of the gas transportation system after finalizing the designin terms of assessing the safety of surrounding people, property, and the environment according to the risk acceptability criteria. The originality of this paper lies in the fact that probabilistic structural integrity assessments of statistical and deterministic-probabilistic structural integrity are combined into one general approach to assessing the reliability of all types of gas storage and transport facilities. The improved methodology helps to understand predicted failure outcomes, defects and damages, effects of aging, permissible and destructive stresses and strains, and other factors relevant to the reliability of pipeline network systems. 


\section{Problem statement}

Pipelines are usually large-capacity pipes that are operated in often challenging conditions, with prolonged incidents resulting in severe environmental and material consequences for the region.

As a rule, gas consumption by industrial and especially household consumers is uneven and fluctuates throughout a day, week, and year. The change in the flow rate is a gradual process because of the system's dynamic behavior. In addition, due to various external factors, such as calendar and weather factors, as well as demographic and economic factors and parameters characterizing the system, market demand tends to change over time [12, 13]. Therefore, gas flow variability is increasing nowadays. Gas pipelines and gas distribution facilities are affected by the technological parameters of their operation. Gas transportation conditions through gas pipelines are transient and have seasonal and daily pumping parameters that cause pressure pulsations. Gas pressure fluctuations contribute to a decline in the output of gas pipelines.

An urgent task in the operation of gas pipeline equipment is to reduce the risk of accidental damage.

Despite the available practical experience in accounting for the potential of errors that ensure industrial safety, as well as the tendency to reduce the rate in accidents at gas pipelines, there are still hazards in the operation of gas pipelines associated with the fire and explosion hazards of transported petroleum products in pipelines near settlements, without excluding risks with catastrophic consequences.

The best protection measures against the effects of dangerous accidents arethe design and construction of hazardous production facilities at a safe distance from objects with people present.

As an example, this paper uses applicable data from an in-service pipeline to further substantiate the estimation process and illustrate the accuracy of the results from the use of the proposed safety parameters.

The study object is the gas distribution network designed to provide consumers with natural gas. The start of the route is the Gas Treatment Unit; the end of the route is the Gas Distribution Plant. The section of the gas transportation system is a high-pressure gas pipeline of category II, length $7.4 \mathrm{~km}, 0.6 \mathrm{MPa}\left(6 \mathrm{kgf} / \mathrm{cm}^{2}\right)$ design pressure and does not belong to the main gas pipeline. Design solutions for safety and risk management in the study area are considered hazardous production facilities and are as follows: the pipeline section(looping) is laid underground and parallel to the pipeline along its entire $7.8 \mathrm{~km}$ route, and the gas storage tank (gas holder) with a capacity of $300 \mathrm{~m}^{3}$ is mounted at the Gas Treatment Unit (Fig. 1). 


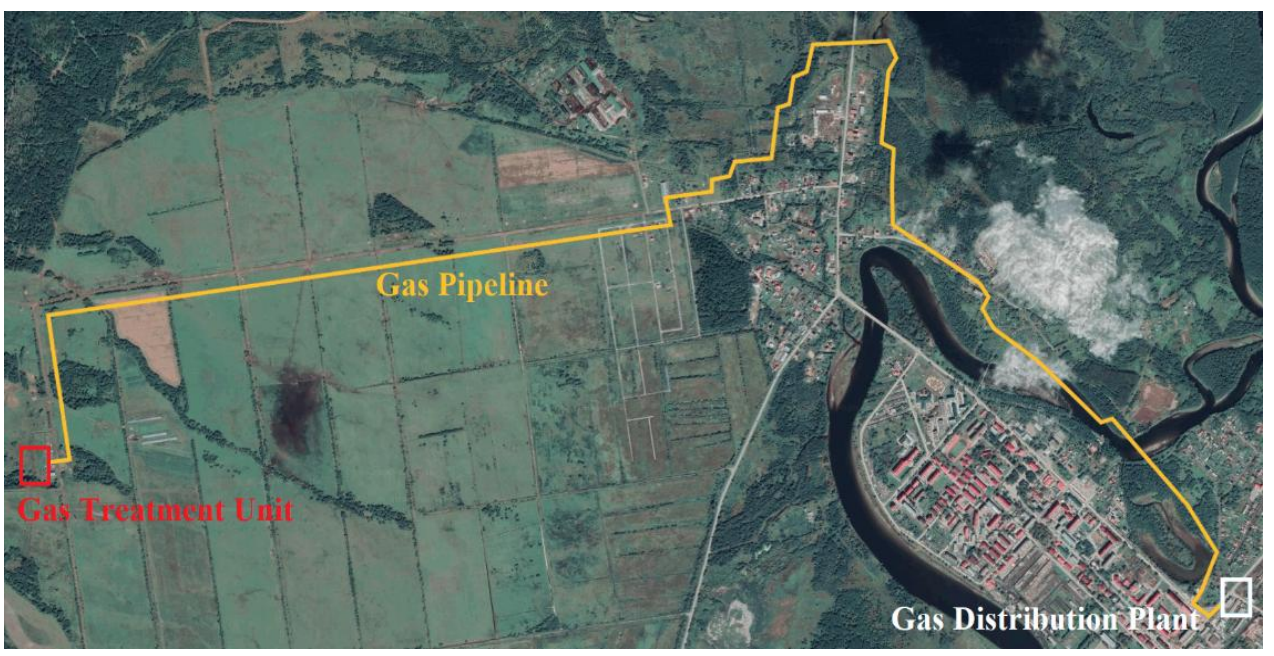

Fig. 1. Site map of the gas transportation system section.

Ensuring the safety of these facilities should include all stages of their design, manufacture, and future operation, with mandatory consideration of both potential accidents and their consequences. However, to achieve greater accuracy in assessing the reliability of the pipeline and the tank, it is advisable to determine the most appropriate safety parameters to be adhered to. In this regard, all risk factors must be considered individually so that parameters can be adjusted for any given pipeline according to the specific conditions.

\section{Methodology of ensuring operability of hazardous production facilities}

The tasks of pipeline gas transportation include finding the optimal solution that could reduce costs with maximum efficiency. It is also necessary to provide data on its ability to maintain the output over a certain period. This object's ability is characterized by reliability and determines its quality.

During operation, the key priority is to ensure equipment reliability to reduce the accident rate. Ensuring uninterrupted and rhythmic gas supply to consumers relies on the efficiency of the gas transmission facilities.

In reality, the reliability of gas supply of pipeline systems is determined by the capacity of gas supply and market demand, which are stochastic at any given moment. Gas supply capacity uncertainty arises from the unpredictable operating state of the system and the dynamic behavior of the pipeline system. Due to the failure and implementation of corrective maintenance in the pipeline system, the uncertainty in the operating state of the system will cause the system to stochastically transit over the mission time to other states. After the transition, the gas flow rate will change accordingly and will affect the capacity of the pipeline system.

The efficiency of the gas supply network is defined as the ratio between the achieved volume of gas supply to all consumers and the estimated demand of all consumers.

The operational efficiency of storage tanks and pipelines should be focused on system diagnostics using the largest databases of risk assessment at industrial plants. Data simulated in scientific laboratories, representing the patterns and 'behavior' of metal deformation, fracture toughness, and rupture, are the basis for the safety assessment of the 
residual resource assignment, the timing of technical analysis, or liquidation of the emergencies and their consequences.

Evaluating the character and size of acceptable or critical defects is essential for estimating the availability of gas supply piping network operations. Cracks can occur during processing or activity. Pipe rupture can occur in cases of critical crack size. It is, therefore, critical that the probability of defects detected during the inspection is calculated and the service life of the pipe and tank is estimated. The lifespan depends on various parameters: crack sizes, properties of the material, and loads. Using these parameters, the lifespan of storage and transport facilities with defects can be determined.

To ensure the reliable operation of the current gas pipelines and for the high-quality design of the new ones, it is important to improve methods for their stress-strain state calculating. One of the effective methods for a technical diagnosis of gas pipelines is the study of their stress-strain state at the sections exposed to dangerous natural and technogenicfactors. Timely information about the existing mechanical stresses in these areas makes it possible to take measures to prevent accidentsand to establish measurement methods for evaluating pipelines on the basis of the defects identified.

Constant-volume gas storage tanks are industrial high-pressure structures that are also in a complex stress-strain state. The stress states of their elements arise already at the stage of manufacturing, construction, and installation. The subsequent increase in the load on the structural elements of the tank is a result of operational stresses, whicheventuallycontributes to the accident risk. Therefore, ensuring reliability and increasing the service life of gas tanks requires a comprehensive consideration of risk reduction, diagnostics, and repair of gas equipment.

Thus, to ensure the reliable operation of hazardous production facilities, the priority is to develop measures to prevent incidents and accidents rather than eradicate their consequences. To create design scheme that adequately describes the state of gas transmission facilities under the action of operational and external influences, it is necessary to analyze their design reliability. It determines the ability to resist external and internal stresses and impacts resultingfrom gas transportation and storage without compromising the integrity of facilities, according to the rules and regulations [14].

The methodology presented below is an improved technique, associated with classical theoretical works, in particular, with many years of studiesconducted by scientists, professors, and the working group under the President of the RAS on the analysis of risks and safety, combining advanced scientific research in the field of industrial safety, the authors of which are N.A.Makhutov, O.V.Aralov, M.M. Gadenin, and others. This methodology can be used to determine the reliability of any pipeline (heating, gas, or oil) or storage tank. There are different reliability metrics (criteria or indicators), but the most commonis the possibilitythe system can perform its function for a given period under specified conditions.

The level of reliability can be measured on the basis of the analysis of emergency failures. Failure is the result of technological hazards in the maintenance of technical facilities. As a rule, accidents with injuries and disasters of different scales that occurred during the operation of gas equipment should be investigated. The study of accidents is an empirical basis for the implementation of technogenic safety in the quantitative assessment of risks.

The most efficient way to reduce the degree of risk for each facilityis to use industrial safety management strategies, where the numerical criteria of 'risk acceptability' are considered, depending on the efficiency of the technological process.

As a rule, damage to hazardous production facilities is measured based on the range of work performed and thus a universal method of comparative risk assessment should be used at each stage of the equipment life cycle. 
Modeling the problems of process analysis and optimizing the conditions for transporting gas are important in pipeline transportation and in particular in the gas industry. Mathematical modeling enables the measurement of technical parameters of gas equipment without carrying out industrial experiments. Besides, production and control processes require special mathematical methods to make scientifically substantiated decisions.

When assessing major risk variables of accidents and disasters, there are three major aspects:

- Risk analysis, i.e., undesirable identification of events, failures, and specific causeconsequence pairings involving the introduction of hazards, as well as the analysis of processes leading to such occurrences

- Risk assessment, i.e.,a quantifying risk procedure

- Risk management as a set of measures aimed at avoiding, minimizing, or decreasing the causes of incidents, i.e., practical activities aimed at risk reduction.

The durability of mechanical components is calculated from a failure/degradation approach or from historical data. In our case, the failure and repair rates of the mechanical components are used as the basis for the calculation of the gas supply reliability. The implementation of risk analysis and monitoring, as well as the development of adverse event scenarios, is focused on the estimation of the operating parameters of hazardous production facilities. In general, the sequence of calculations is as follows: 'strength $R_{\sigma}(\tau)-$ rigidity $R_{\delta}(\tau)$ - stability $R_{\lambda}(\tau)$ - resource $R_{N \tau}(\tau)$ - reliability $P_{Q R}(\tau)$ - survivability $L_{l d}(\tau)$ safety $S(\tau)$ - risk $R(\tau)$ - protect ability $Z_{K}(\tau)^{\prime}$. All parameters are functions of time $\tau$ at all stages of their service life cycle.

The general formulation of the research questions boils down to resistance to any future failure mechanisms. There is a wide range of mitigation and support inspection techniques available. They allow to foresee technical conditions in the design, operation, and maintenance of hazardous production facilities.

The risk assessment components apply to all hazards. Since industrial facilities are analyzed, it is necessary, firstly, to analyze the probabilities of occurrence of hazardous situations, and secondly, to analyze the consequences of hazard implementation.

The key and appropriate indicator for determining the reliability of gas pipelines and onshore gas storage tanks is the likelihood of a non-failure operation

$$
P(\tau)=e^{-\lambda \tau}
$$

Where $\lambda=$ failure rate; $\tau=$ time.

The key statistical feature of accidents in pipelinesand tanks is the severity of accidents, estimated as 'events per time and distance'

$$
\lambda=\frac{N}{L \cdot \tau}
$$

Where $L=$ length of gas pipeline; $\tau=$ time.

To assess the severity of emergencies (including those resulting from employee errors), accident statistics or calculated data on the reliability of technological equipment unique to the affected production facility, collected from similar functional items, can also be used.

Protect ability $Z_{\kappa}(\tau)$ is defined through emerging $R(\tau)$ and acceptable $[R(\tau)]$ risks as

$$
Z_{\kappa}(\tau)=R(\tau) \cdot\left(1-\frac{[R(\tau)]}{R(\tau)}\right)
$$


The protect ability level determines all the main requirements: safety $S(\tau)$, risks $R(\tau)$, resource $R_{N \tau}(\tau)$, reliability $P_{Q T}(\tau)$, survivability $L_{l d}(\tau)$, strength $R_{\sigma}(\tau)$, rigidity $R_{\delta}(\tau)$, and stability $R_{\lambda}(\tau)$ [17].

Risks formed at this lifecycle stage $\tau$ are assessed by probability $P(\tau)$ of failures, emergency or catastrophic situations and their losses $U(\tau)$ as

$$
R(\tau)=P(\tau) \cdot U(\tau)
$$

The risk function is more preferable for assessing the safety of the structure, as it requires an assessment of potential losses.

Quantitative risk assessment analyzes potential failure scenarios and calculates the range of possible losses from each failure scenario. The mathematical approach to risk quantification involves scenario models such as event trees and fault trees, which are commonly used today.

These scenarios related to potential human error during the life cycle of hazardous production facilities that could directly threaten integrity should supplement the event trees/fault trees that represent possible scenarios in relation to pipeline risk factors.

Human errors occur at different stages of the project and persist over the entire life cycle of the hazardous production facilities and should be integrated into risk calculations. Moreover, risk assessment linked to human error rates should include scenarios, where there is a chance in the probability distributions of load-bearing capacities and external loads, as well as limiting states due to a large number of error probabilities [10].

Developing emergency scenarios make it possible to collect source data on possible impacts and adverse factors, as well as to provide a framework for evaluating the risk of hazardous production facilities.

Acceptable risks $[R(\tau)]$ based on the analysis of critical failures, accidents, and catastrophes are assigned according to the most dangerous, critical parameters $P_{k}(\tau), U_{k}(\tau)$, $R_{k}(\tau)$, with the introduction of risk margin $n_{R}\left(n_{R} \geq 1\right)$

$$
[R(\tau)]=\frac{R_{\kappa}(\tau)}{n_{R}}=\frac{P_{\kappa}(\tau) \cdot U_{\kappa}(\tau)}{n_{R}} .
$$

The assessment of acceptable risk requiressetting priorities for activities that can successfully improve safety, rather than principles that shape the goals ofsafetythat must be met.

Ensuring safety $S(\tau)$ byusing the risk function is to satisfy the following condition:

$$
S(\tau)>0 \text { as long as } R(\tau) \leq[R(\tau)] .
$$

The difficulty in enforcing condition(6) for the measurement of gas pipelines and storage tanks is due to the need for probabilistic models of foreseeable loads, stresses, and component strengths. It is also important to create an appropriate risk $[R(\tau)]$ level that can be quite troublesome nowadays due to the lack of available loss statistics.

Quantitatively, survivability is measured by comparing the development parameters of defects $l(\tau)$ and the damage $d(\tau)$

$$
l(\tau) \leq[l(\tau)] ; d(\tau) \leq[d(\tau)] ; \quad[l(\tau)]=\frac{l_{\kappa}(\tau)}{n_{l}} ; \quad[d(\tau)]=\frac{d_{\kappa}(\tau)}{n_{d}},
$$

where $[l(\tau)],[d(\tau)]=$ allowed defects and damages; $n_{l}, n_{d}=$ margins for defects and damages as long as $\left\{n_{l}, n_{d}\right\} \geq 1$.

Reliability $P_{Q R}(\tau)$ is defined by the probabilistic characteristics for which the event trees or the fault trees are often widely used. 
Event tree analysis is a set of quantitative or qualitative methods used to classify potential outcomes and probabilities of the initiation event. In our case, the logical event tree method is used for objects defined by the features of the improved gas transmission project, which helps to reduce the accident rate and allow the identification of sequences of events which in their turn leadto the appearance of certain consequences of the initial event.

To classify potential scenarios for the occurrence of hazardous incidents and the development of field pipelines and gas storage tanks, the following types of accidents must be taken into account: torch; flash fire (delayed ignition); explosion (delayed ignition); straight fire (delayed ignition on liquid phase expiration after outbreak and fire explosion).

It is also necessary to consider the frequency of depressurization, failure, and collapse of the pressure vessel for all the leakage sizes defined for the technical equipment block [15].

A Fault tree is a graphical representation of conditions or other factors causing an unexpected occurrence called a peak event. Fault tree analysis is a deductive method of analysis designed to determine the cause or combination of causes of events.

Risk management techniques are generally quantitative. However, a full quantitative analysis is not always possible due to a lack of knowledge about the operation of gas transportation systems. At the same time, risk management assessment means taking into account the most significant possible accidents in the operation of such facilities. Therefore, a comparative quantitative or qualitative risk ranking can be useful in such a situation.

Technical risk is defined as

$$
R_{T}(\tau)=1-P_{Q R}(\tau)
$$

Reliability may include dependability, durability, maintainability, retentively, or their various combinations, depending on the purpose and operating conditions at the facility [16].

The criteria of damage $U(\tau)$, mainly economic, which correspond to the conditions under consideration(failures, accidents, and catastrophes) are defined in the sense of the reliability $P_{Q T}(\tau)$ and technological risk $R_{t}(\tau)$ analysis.

The load-bearing resource $R_{N t}$ is defined by normal and refined calculations of cyclic $(N)$ and long-term strength $(\tau)$ for nominal and local stresses and strains $\sigma_{\alpha n}(\tau), \sigma_{\alpha \max }(\tau), e_{\alpha n}(\tau)$, $e_{\alpha \max }(\tau)$ as

$R_{N \tau}=F_{N \tau}\left\{\left(\sigma_{a n}(\tau), \sigma_{a \max }(\tau)\right),\left(e_{a n}(\tau), e_{a \max }(\tau)\right),\left(\left(" \sigma_{a}-N "\right),\left(" e_{a}-N^{\prime \prime}\right)\right),\left((" \sigma-\tau "),\left(" e-\tau^{\prime \prime}\right)\right)\right\}$

where ' $\sigma_{\alpha}-N$ ', ' $e_{\alpha}-N$ ', ' $\sigma-\tau$ ', ' $e-\tau$ ' are equations of cyclic $(N)$ and long-term strength $(\tau)$ curves in stresses and strains obtained from standard tests of laboratory samples.

The rigidity of the bearing elements $R_{\delta}(\tau)$ is calculated by the measurement of their displacements $\delta(\tau)$ under the operational influences $Q^{\top}(\tau)$ and the selected cross-sectional size $F$ and length $L$ as

$$
R_{\delta}(\tau)=\delta(\tau) \leq\left[R_{\delta}(\tau)\right]=\frac{\delta_{\mathrm{\kappa}}(\tau)}{n_{\delta}},
$$

where $R_{\delta}(\tau)=$ permissible deformations; $\delta_{k}(\tau)=$ critical deformations; $n_{\delta}=$ margin for deformation $\left(n_{\delta} \geq 1\right)$.

Stability $R_{\lambda}(\tau)$ is determined by the loss of stability

$$
R_{\lambda}=\frac{Q_{\mathrm{K}}(\tau)}{Q^{\ni}(\tau)}=n_{y}
$$


where $Q_{k}(\tau)=$ critical loads during the loss of stability; $n_{y}=$ margin for stability $\left(n_{y} \geq 1\right)$.

The operational history of hazardous production facilities suggests that the execution of serious accident scenarios is, as a rule, correlated with the inability to maintain the strength of structural elements of hazardous production facilities.

Strength $R_{\sigma}(\tau)$ of load-bearing elements is calculated using the overall nominal and local strain $\sigma_{\max }(\tau)$ stresses $e_{\text {amax }}(\tau)$ for extreme operating loads $Q_{\max }(\tau)$ as

$$
\left.\sigma_{\max }(\tau), e_{\max }(\tau)\right\} \leq\{[\sigma(\tau)],[e(\tau)]\}=\left\{\frac{\sigma_{\mathrm{\kappa}}(\tau)}{n_{\sigma}}, \frac{e_{\mathrm{\kappa}}(\tau)}{n_{e}}\right\},
$$

where $[\sigma(\tau)],[e(\tau)], \sigma_{k}(\tau), e_{k}(\tau)$ are the permissible and destructive stresses and strains for given loading conditions (by the number of cycles $N$, time $\tau$ and temperature $t$ ); $n_{\sigma}, n_{e}=$ margins for stress and strain $\left(1 \leq n_{\delta} \ll n_{e}\right)$.

Modern estimations of the strength and resources of hazardous production facilities represent a new approach in managing their performance. This can be accomplished by scientifically substantiated use of linear and nonlinear deformation and fracture methods, risk analysis, justification of resources for safe operation, and prevention of accidents [14].

Set of equations (3) - (12) consists of the main dependencies required for the computational and experimental analysis and the determination of the key parameters of safety, risks, and security of operated and designed facilities of the gas transportation systems $[18,19]$. The basis set of requirements for the strength, resource, and safety of tanks and pipelines should include the consistent application of these ratios with given structural, technological, and operational parameters.

Continuous reliability control reduces the likelihood of emergencies at hazardous production facilities that do not meet the real conditions, thereby increasing the overall reliability of the gas pipeline system.

\section{Conclusion}

The proposed combined algorithm, based on classical theoretical works of scientists of the Russian Academy of Sciences and other Russian research institutes in the field of safety and emergencies, describes the state of the objects under the impact of operational and external loads is necessary for the determination and evaluation analysis of technogenic safety parameters (strength, rigidity, stability, resource, reliability, survivability, risk, and protect ability) of the gas transportation system without carrying out industrial experiments.

The presented methodology ensures scientific and effective management of natural gas transportation and storage facilities, maintains the technical conditions of hazardous production facilities during the initial and final periods of their operation.

\section{References}

1. W.Yu, S.Song, Y.Li, Y.Min, W. Huang, K.Wen, J. Gong. Gas supply reliability assessment of natural gas transmission pipeline systems. Energy 162: 853-870(2018)

2. K.A.Pambour, B.C.Erdener, R.Bolado-Lavin, G.P.J. Dijkema, SAInt - A novel quasidynamic model for assessing security of supply in coupled gas and electricity transmission networks. Applied energy, 203: 829-857(2017).

3. W.Yu, K.Wen, Y.Min, L.He, W.Huang, J. Gong, A methodology to quantify the gas supply capacity of natural gas transmission pipeline system using reliability theory. Reliability Engineering \& System Safety, 175: 128-141 (2018). 
4. S.Clegg, P. Mancarella, Integrated electrical and gas network flexibility assessment in low-carbon multi-energy systems. IEEE Transactions on Sustainable Energy, 7(2): 718-731(2015).

5. V.A.Zhila, O.V.Aleksyutina, D.A. Santalov, Effect of underground pipelines corrosion damage on the reliability of gas supply systems. Journal of Plumbing, Heating and Air Conditioning, 94, 22-33 (2005)

6. V. N. Medvedev, et al. About the causes of main gas pipelines accidents.Aging problems of steel of main pipelines: 110-121 (2006).

7. A.V.Popov, A.L. Saruev, Structure and properties of the main gas pipelines metal after 30 years of operation. Chemistry of oil and gas: 515-522 (2003)

8. Y.Sun, L.Ma, J. Morris, A practical approach for reliability prediction of pipeline systems. European Journal of Operational Research: 210-214 (2009).

9. A.Amirat, A.Mohamed-Chateauneuf, K. Chaoui, Reliability assessment of underground pipelines under the combined effect of active corrosion and residual stress. International Journal of Pressure Vessels and Piping, 83(2): 107-117 (2006).

10. N.A.Makhutov, D.O. Reznikov, Consideration of Threats Associated with the Human Factor when Assessing the Security of Hazardous Production Facilities. Occupational Safety in Industry: 60-67(2015).

11. S.Rimkevicius, A.Kaliatka, M.Valincius, G. Dundulis, Development of an approach for reliability assessment of pipeline network systems. Applied energy: 22-33 (2012).

12. J. Szoplik, Forecasting of natural gas consumption with artificial neural networks. Energy: 208-220(2015)

13. M.Thaler, I.Grabec, A. Poredoš, Prediction of energy consumption and risk of excess demand in a distribution system. Physica A: Statistical mechanics and its applications355(1): 46-53(2005)

14. N.A. Makhutov, Structural strength, resource and anthropogenic safety: in 2 parts. Part 1: Criteria of strength and resource; Part 2: Substantiation of resource and safety. (Novosibirsk: Nauka,2005).

15. W. Kent Muhlbauer, Pipeline Risk Assessment: The Definitive Approach and Its Role in Risk Management. (Austin: Expert Publishing, LLC,2015).

16. O.V. Aralov, Quality management methodology for complex engineering systems in major oil and oil product pipelines. Science \& Technologies: Oil and Oil Products Pipeline Transportation9(6): 608-625 (2019).

17. N.A.Makhutov, N.V.Abrosimov, M.M. Gadenin, Provision of Safety - The Priority in The Sphere of Fundamental and Applied Research. Economic and Social Changes: Facts, Trends, Forecast3(27): 39-61(2013).

18. N.A.Makhutov, M.M. Gadenin, Fundamental regularities of technogenic safety in feasibility substantiation of future gas-transport systems. VestiGazovoyNauki2(34): 109-124(2018).

19. N.A.Makhutov, M.M.Gadenin, A.S.Pecherkin, B.A. Krasnyh, Calculation and experimental approaches to the analysis and provision of the service life and safe operation life of industrial facilities. Occupational Safety in Industry: 7-15 (2020). 